\title{
Transfer of Carbon Dioxide within Cultures of Microalgae: Plain Bubbling versus Hollow-Fiber Modules
}

\author{
Ana P. Carvalho and F. Xavier Malcata* \\ Escola Superior de Biotecnologia, Universidade Católica Portuguesa, Rua Dr. António Bernardino de Almeida, \\ P-4200-072 Porto, Portugal
}

\begin{abstract}
In attempts to improve the metabolic efficiency in closed photosynthetic reactors, availability of light and $\mathrm{CO}_{2}$ are often considered as limiting factors, as they are difficult to control in a culture. The carbon source is usually provided via bubbling of $\mathrm{CO}_{2}$ enriched air into the culture medium; however, this procedure is not particularly effective in terms of mass transfer. Besides, it leads to considerable waste of that gas to the open atmosphere, which adds to operation costs. Increase in the interfacial area of contact available for gas exchange via use of membranes might be a useful alternative; microporous membranes, in hollow-fiber form, were tested accordingly. Two hollow-fiber modules, different in both hydrophilicity and outer surface area, were tested and duly compared, in terms of mass transfer, versus traditional plain bubbling. Overall volumetric coefficients $\left(\mathrm{K}_{\mathrm{L}} \mathrm{a}\right)$ for $\mathrm{CO}_{2}$ transfer were $1.48 \times 10^{-2} \mathrm{~min}^{-1}$ for the hydrophobic membrane, $1.33 \times 10^{-2} \mathrm{~min}^{-1}$ for the hydrophilic membrane, and $7.0 \times$ $10^{-3} \mathrm{~min}^{-1}$ for plain bubbling. A model microalga, viz. Nannochloropsis sp., was cultivated using the two aforementioned membrane systems and plain bubbling. The produced data showed slight (but hardly significant) increases in biomass productivity when the hollow-fiber devices were used. However, hollow-fiber modules allow recirculation of unused $\mathrm{CO}_{2}$, thus reducing feedstock costs. F urthermore, such indirect way of supplying $\mathrm{CO}_{2}$ offers the additional possibility for use of lower gas pressures, as no need to counterbalance hydrostatic heads exists.
\end{abstract}

\section{Introduction}

In economic terms, microalgae can be seen as microorganisms capable of producing highly valuable compounds (e.g., natural pigments and polyunsaturated fatty acids) starting from inexpensive resources (Carval ho and Malcata, 2000).

However, to maximize productivities, microal gae cultures must be carefully controlled for contamination, light supply, temperature, $\mathrm{pH}$ and nutrient profile, including gaseous substrates. Within this set of environmental conditions, light and $\mathrm{CO}_{2}$ supply are key processing parameters, especially owing to difficulties associated with their control.

Since the $\mathrm{CO}_{2}$ available for transfer from the growth medium to the cell cytoplasm must previously cross the interface between the gas phase and the liquid growth medium phase, the amount and qual ity of such interface is rather important. There are basically two ways to increase said interfacial area, which can be described as passive and active modes (Becker, 1994). In the passive mode, extensive air/liquid interface areas are employed, and the gas is transported to theliquid by plain diffusion. An example, on the industrial scale, is the cultivation of Dunaliella sp. in Australia in natural lakes ca. 50-300 ha large but only a few centimeters deep. The active mode takes advantage of extra apparata for aeration, either via injection of the gas in the medium or via spraying of the medium in the gas, both under turbulent regimes (Becker, 1994). Note that $\mathrm{CO}_{2}$ in the air accounts for a

* xmalcata@esb.ucp.pt mere $0.03 \%(\mathrm{v} / \mathrm{v})$, so either of those modes leads to poor transport rates of $\mathrm{CO}_{2}$; to overcome this gradient constraint, $\mathrm{CO}_{2}$-enriched air is commonly employed.

Recalling the active mode of mass transfer, bubbling of $\mathrm{CO}_{2}$-enriched air is usually performed via sintered porous stones, where tiny holes were drilled; such parameters as bubble size, gas flow rate and $\mathrm{CO}_{2}$ pressure can then be adjusted to meet the culture requirements (Talbot et al., 1991). Unfortunately, two major drawbacks often arise: (i) the gas transfer devices do rapidly get fouled, which requires frequent cleaning; and (ii) most $\mathrm{CO}_{2}$ is eventually lost to the atmosphere, which leads to increases in operation costs as pure $\mathrm{CO}_{2}$ is an expensive utility (Tapie, 1988). The installation of covered sparging-diffusion systems, aimed at trapping the bubbles under (transparent) plastic sheets, leads to increases in the gas-liquid contact time (Heussler et al., 1978), but it is unable to completely solve the problem because considerable amounts of $\mathrm{CO}_{2}$ are still lost to the atmosphere at the ends of the plastic sheets.

E nlarged areas of contact between $\mathrm{CO}_{2}$ and the culture medium may be achieved by diffusing the pure gas through membranes of silicone tubing (Kun-Lee and K wan-Hing, 1989), which is permeable to $\mathrm{CO}_{2}$; according to these authors, said procedure offers several advantages when compared with plain bubbling, viz., drastic reduction of $\mathrm{CO}_{2}$ losses to the atmosphere, possibility to accurately control $\mathrm{CO}_{2}$ transfer rates and no need for concentrated gaseous mixtures of $\mathrm{CO}_{2}$ for efficient transfer. However, the procedure suffers from severe impairments: (i) silicon membranes are quite expensive, and since the transfer flow rate is proportional to the inter- 


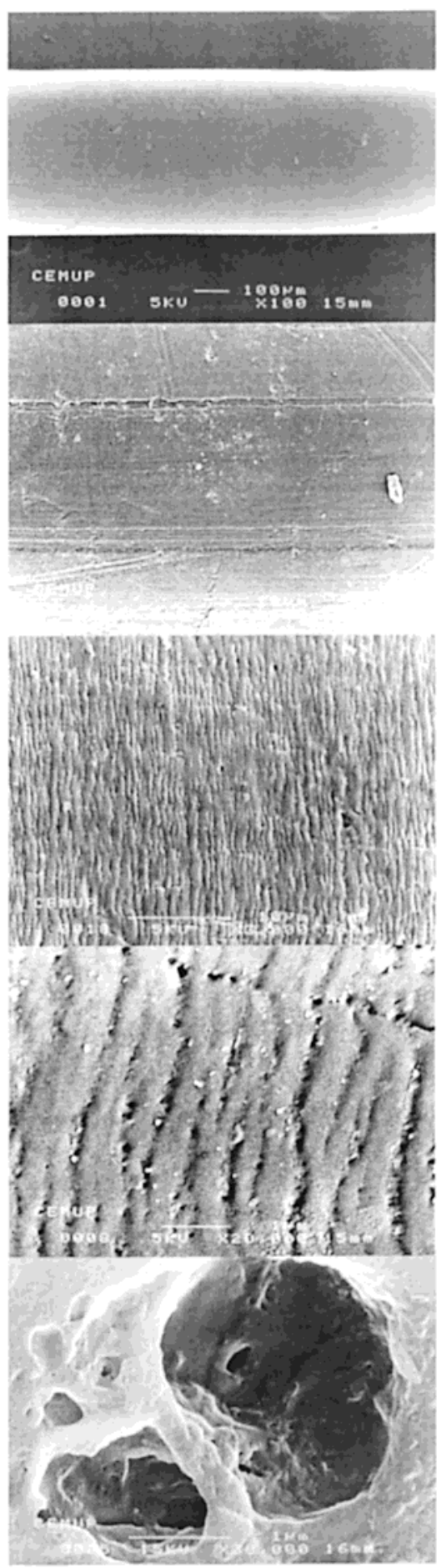

Figure 1. Scanning electron micrograph of a single hydrophobic fiber (100x, 400x, 3000x, 20000x, and 30000x magnifications).

facial area, large membranes are necessary; and (ii) high internal pressures are required if economical transfer rates are to be achieved (as in bubbling), which implies the use of thick membrane walls that in turn create an extra resistance to gas transfer.

In conclusion, the overall performance associated with any of the aforementioned techniques employed at present to supply $\mathrm{CO}_{2}$ to al gal cells is still not satisfactory from an engineering point of view. An alternative possibility relies on the (so far) scarcely explored potential of microporous hollow-fiber membranes, i.e., bundles of polymeric porous fibers, potted to inlet/outlet ports in their ends, and contained in appropriate housings. Commercial fiber modules are built from tiny hollow fibers, with typical internal diameters of ca. $250 \mu \mathrm{m}$; their permeability, which depends obviously on the construction material, is depicted in Figure 1 . As a result of the enormous number of fibers insi de each module, the ratio between the membrane outer area and the external dimensions is quite high.

The mass transfer flux increases with the transmembrane pressure and the recirculation flow rate. Although raises in flow rate and pressure would increase the mass flux, pressures are upperly constrained by the mechanical limits of the equipment (typically $40 \mathrm{psi}$ ). Hence, the fluxes are preferably increased via increases in the recirculation flow rate, while the inlet pressure is maintained close to the maximum allowed. Use of hollow-fiber devices has been reported in the culture of mammalian cells (Gallagher et al., 1987), in the culture of the microal ga Chlamydomonas reinhardtii (Chen and J ohns, 1995), in continuous culturing of the microalga Phaeodactylum tricornutum in seawater (Marsot et al., 1992) and in the culture of the cyanobacterium Anabaena variabilis (Markov et al., 1995).

The objective of this study was to compare the performance, in terms of mass transfer, between plain bubbling of $\mathrm{CO}_{2}$ in a liquid medium and recirculation of $\mathrm{CO}_{2}$ inside two hollow-fiber modules of distinct nature, using phenomenological approaches. Such comparison was based on the overall volumetric coefficients of transfer of $\mathrm{CO}_{2}$ $\left(K_{L} a\right)$ in those three situations. The growth of the microal ga Nannochloropsis sp. was considered as a model system, and supply of $\mathrm{CO}_{2}$ was via either membranemediated transfer or direct bubbling.

\section{Theoretical Considerations}

The overall resistance to transfer of $\mathrm{CO}_{2}$ from the bulk gas to the interior of the cells can be conceptually calculated via the sum of several individual resistances to mass transfer. According to the double-film theory, those resistances are located (i) in each of the thin layers adjacent to the interface between the gas and the liquid phases, (ii) within the separating membrane and (iii) in the vicinity of the surface of the cells themselves. However, even in dense cell cultures, the resistance at the gas-liquid interface is much larger than at the boundary layer of the cells, because very small values (ca. $30 \mathrm{ppm}$ ) of $\mathrm{CO}_{2}$ on the cell surface are sufficient to avoid limitation upon photosynthesis (Goldman et al., 1981; Becker, 1994). Hence, the overall resistance to $\mathrm{CO}_{2}$ transfer from the gas to the cells $\left(1 / K_{L}\right)$ is essentially restricted to the sum of resistances imposed by the gas boundary layer $\left(1 / k_{g}\right)$, the membrane material $\left(1 / k_{m}\right)$, and the liquid boundary layer $\left(1 / k_{\mathrm{L}}\right)$, which can be equated by

$$
\frac{1}{\mathrm{~K}_{\mathrm{L}}}=\frac{1}{\mathrm{Hk}_{\mathrm{g}}}+\frac{1}{\mathrm{HK}_{\mathrm{m}}}+\frac{1}{\mathrm{~K}_{\mathrm{L}}}
$$

where $K_{L}$ is an overall mass transfer coefficient based on the liquid-side conditions, and $\mathrm{k}_{\mathrm{g}}, \mathrm{k}_{\mathrm{m}}$ and $\mathrm{k}_{\mathrm{L}}$ are mass transfer coefficients in the gas film, within the membrane and in the liquid film, respectively. It may be argued that in the case of a hydrophilic system, where the pores of the membrane would be filled with the liquid culture medium, an extra resistance term would be necessary so as to account for the resistance brought about by molecular transport inside the pores filled with stagnant liquid (i.e., $1 / \mathrm{K}_{\mathrm{L} \text {, pore }}$ ). However, an order of magnitude analysis of the time scale associated with diffusion inside the pores (i.e., $\delta^{2} / \mathrm{D}_{\mathrm{L}}$, where $\delta$ is the radius of the pore and $\mathrm{D}_{\mathrm{L}}$ is the diffusivity of $\mathrm{CO}_{2}$ in water) and the time scale associated with diffusion through the liquid side boundary layer $\left(\epsilon^{2} / D_{L}\right.$, where $\epsilon$ is the thickness 
of the stagnant liquid film) indicated that such extra resistance term can be neglected (because $\delta \approx 10^{-7} \mathrm{~m}$ and $\epsilon>10^{-5} \mathrm{~m}$ ).

Since $\mathrm{CO}_{2}$ is poorly soluble in water, Henry's constant is large (e.g., $\mathrm{H}_{\mathrm{CO}_{2} / \mathrm{H}_{2} \mathrm{O}}=170 \mathrm{MPa}$, at $25^{\circ} \mathrm{C}$ ) (Breck et al ., 1989). Furthermore, $k_{m} \approx\left(D_{\text {eff }} / \xi\right)$ (where $D_{\text {eff }}$ is the effective diffusivity within the porous membrane and $\xi$ is the thickness of the membrane). It is known that typically $D_{\text {eff }}<D_{\llcorner} / 10$, owing to pore tortuosity and fractional porosity, so $1 / \mathrm{Hk}_{\mathrm{m}} \approx 1 / 10 \mathrm{H} \mathrm{k}_{\mathrm{L}}$ for the hydrophilic case, and $1 / \mathrm{Hk}_{\mathrm{m}} \approx 1 / 10 \mathrm{H}_{\mathrm{g}}$ for the hydrophobic case; hence, the contribution of $1 / \mathrm{Hk}_{\mathrm{m}}$ to the overall resistance can be neglected in either case.

Finally, the diffusivity in gaseous phases is ca. 4 orders of magnitude larger than the diffusivity in liquid phases (for similar degrees of stirring, as is the case), so the liquid boundary layer accounts for most resistance to mass transfer. Under these realizations, eq 1 becomes simply

$$
\frac{1}{\mathrm{~K}_{\mathrm{L}}} \cong \frac{1}{\mathrm{~K}_{\mathrm{L}}}
$$

The total amount of $\mathrm{CO}_{2}$ transferred from the gaseous to the liquid phase can then be calculated via the following simple mass balance

$$
\frac{d C}{d t}=K_{L} a\left(C^{*}-C\right)
$$

where a denotes the gas-liquid interfacial area per unit volume of liquid and $\mathrm{K}_{\mathrm{L}}$ a denotes, as usual, the overall volumetric mass transfer coefficient of $\mathrm{CO}_{2} ; \mathrm{C}$ is the concentration of $\mathrm{CO}_{2}$ dissolved in the bulk liquid and $\mathrm{C}^{*}$ is the concentration of $\mathrm{CO}_{2}$ that would be dissolved if the liquid phase were saturated in this gas. Assuming $C^{*}$ as constant (because temperature is kept constant) and $\mathrm{K}_{\mathrm{L}} \mathrm{a}$ as independent of $\mathrm{C}$, integration of the former equation from the lower limit $\mathrm{C}=0$ at $\mathrm{t}=0$ (because the starting liquid phase is free of $\mathrm{CO}_{2}$ ) leads to

$$
\mathrm{C}=\mathrm{C} *\left(1-\mathrm{e}^{-\mathrm{K}_{\mathrm{L}} \mathrm{at}}\right)
$$

The values of $C$ are indirectly calculated, on the basis of the $\mathrm{pH}$ values measured in the liquid phase throughout the time frame of the experiments, according to the carbonic acid dissociation equilibrium relationships (Ferreira et al., 1998). The parameter $K_{L} a$ in eq 4 is then fitted to the experimental values of $\mathrm{C}$ using nonlinear regression analysis.

\section{Materials and Methods}

Measurement of Mass Transfer Rates. The setup used for mass transfer measurements is depicted in Figure 2. A solution of $0.01 \mathrm{M}$ sodium hydroxide was poured into a closed 1-L glass vessel of $10.8 \mathrm{~cm}$ diameter, magnetically stirred to avoid buildup of concentration gradients, and recirculated at $0.55 \mathrm{~mL} / \mathrm{s}$ through the lumen of the hollow-fiber device using a peristaltic pump (Ismatec, Switzerland). A mixture of air with $1 \%(\mathrm{v} / \mathrm{v})$ $\mathrm{CO}_{2}$ was generated by adequate continuous mixing of air with pure $\mathrm{CO}_{2}$, with flow rates duly monitored by flowmeters (Aal borg); the mixture was circulated at 150 $\mathrm{mL} / \mathrm{min}$ through the shell side of the hollow fibers. Two different hollow-fiber modules were used: (i) a hydrophobic membrane, from I NTE RSEP (UK), model 5PCM104, made of extended polypropylene fibers with $0.2 \mu \mathrm{m}$ average pore diameter, $30.4 \mathrm{~cm}$ length and $240 \mu \mathrm{m}$ fiber internal diameter, with ca. 7400 fibers and a total

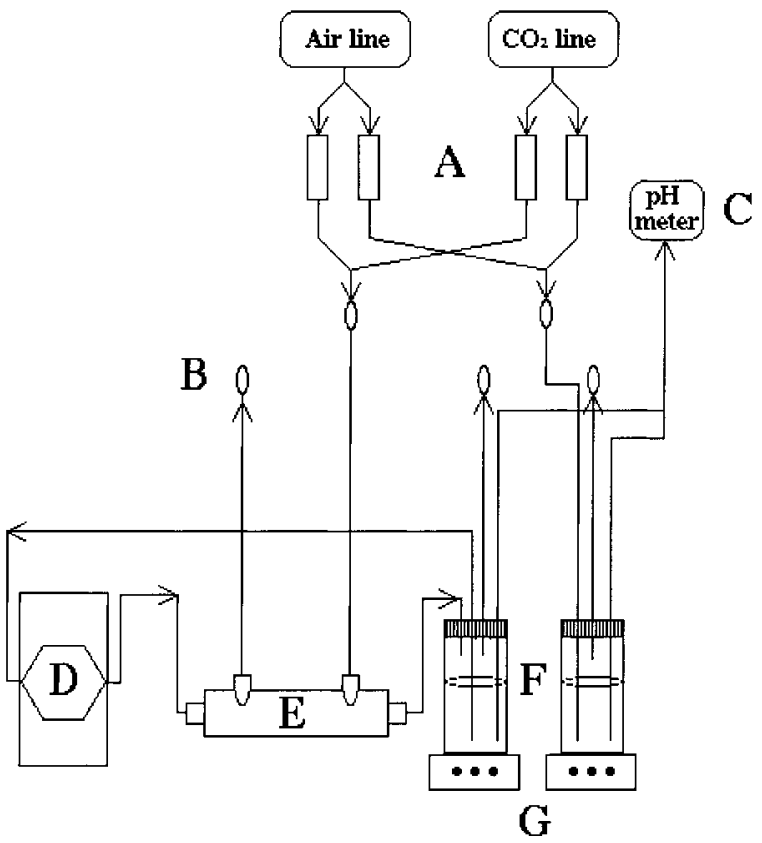

Figure 2. Setup used for the experimental determinations of parameter $\mathrm{K}_{\mathrm{L}} \mathrm{a}$ : A, flowmeters; B, gas filters; C, pH meter; D, peristaltic pump; $E$, hollow-fiber module; $F$, stirred flask containing the $\mathrm{NaOH}$ solution; and $\mathrm{G}$, stirring devices.

effective area of $1.7 \mathrm{~m}^{2}$; and (ii) a hydrophilic membrane, from $A / G$ Technology (US), made of polysulfone fibers with $0.2 \mu \mathrm{m}$ average pore size, $31.5 \mathrm{~cm}$ length and 1000 $\mu \mathrm{m}$ fiber internal diameter, with ca. 140 fibers and a total effective area of $0.14 \mathrm{~m}^{2}$. The measurements of $\mathrm{pH}$ were periodically made using a standard $\mathrm{pH}$ meter (Ingold, UK). The method of determining the $\mathrm{CO}_{2}$ concentration has been described elsewhere (Ferreira et al., 1998).

A second 1-L glass vessel was set up and subjected to si milar conditions, but in the absence of the hollow-fiber device. In this control setup, the supply of $\mathrm{CO}_{2}$ was by direct bubbling through a glass tube of $6-\mathrm{mm}$ internal diameter, at the bottom of the glass reservoir. This system was coupled to two independent flowmeters, so as to guarantee the same flux of the gaseous mixture, irrespective of overall working pressure and topical resistances.

Performance of Culture of Microalgae. Nannochloropsis sp. (NAN NO-2) was a gift from I nstituto $\mathrm{N}$ acional de Engenharia e Tecnologia Industrial (Lisbon, Portugal).

A 2-L glass flask was connected to the hollow-fiber module and was used for the autotrophic cultivation of the model microalga (Figure 3 ). Cells were grown in an artificial seawater medium, ASW (Borowitzka, 1988), buffered with glycylglycine, thermostated at $20 \pm 0.5^{\circ} \mathrm{C}$ by a water bath, and subject to a sustained continuous light cycle, provided by a bank of 12 cold fluorescent lamps equally spaced along the culture vessels. The $\mathrm{pH}$ of the culture was adjusted to ca. 8.0 before autoclave sterilization. Agitation was obtained by stirring the culture vessels with Teflon-coated magnetic stirring bars.

The cul ture was recirculated through the lumen of the hollow fibers by a peristaltic pump (Ismatec) at $26 \mathrm{~mL} /$ min. A mixture of air with $1 \%, 5 \%$ or $10 \%(\mathrm{v} / \mathrm{v}) \mathrm{CO}_{2}$ was obtained via mixing the two gases in the required proportions, as monitored by two flowmeters. The mixture was humidified in washing gas flasks and filtered before entering the system, where it was circulated at $150 \mathrm{~mL} / \mathrm{min}$ through the shell side of the hollow fibers. Samples were collected in 100-mL glass flasks (previously sterilized), sealed with a cotton plug and connected to 


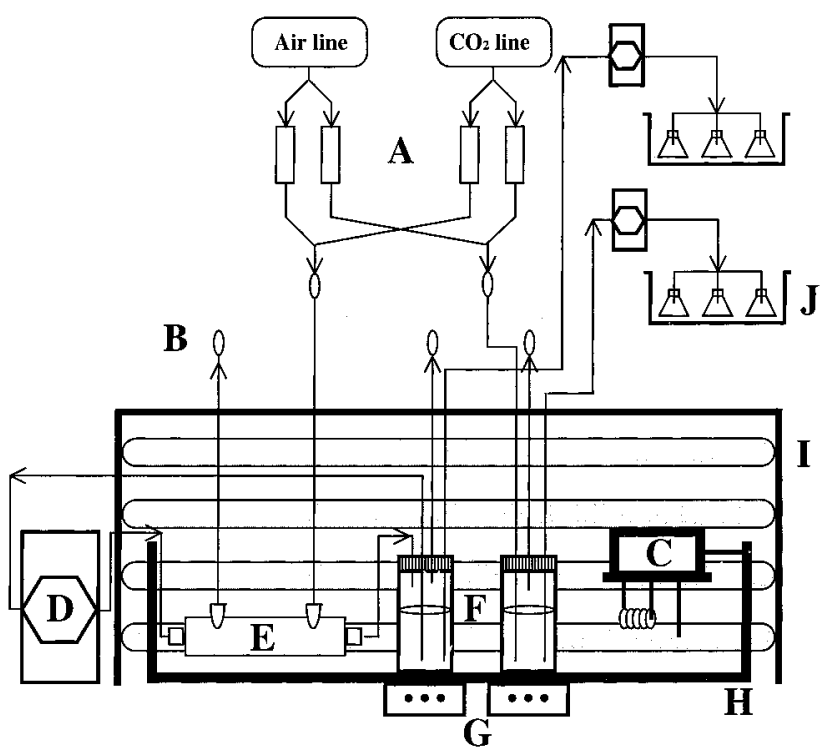

Figure 3. Setup used for the experimental growth of Nannochl oropsis sp.: A, flowmeters; $\mathrm{B}$, gas filters; $\mathrm{C}$, temperature controller; D, peristaltic pump; E, hollow-fiber module; $F$, stirred flask containing the microalgae culture; $\mathrm{G}$, stirring devices; $\mathrm{H}$, water bath; I, illuminated system; and J , sample recovery flasks.

the 2-L flask by plastic tubing; the recovery of each sample from the main flask was performed with a second peristaltic pump (Masterflex, US). The two membrane cartridges (i.e., hydrophobic and hydrophilic) were tested under similar operating conditions.

A second 2-L flask was cultivated under identical environmental conditions, but without connection to the hollow-fiber module.

Ash free dry weight (AFDW) was determined by filtering $10 \mathrm{~mL}$ of culture through preconditioned Whatman GF/C glass fiber filters (UK), drying at $105^{\circ} \mathrm{C}$ to constant weight, and heating at $550^{\circ} \mathrm{C}$ for $1 \mathrm{~h}$. Optical microscope observations were performed with an Olympus $\mathrm{CH}-2$ microscope (J apan).

\section{Results and Discussion}

Mass Transfer Parameters. When equal amounts of gaseous $\mathrm{CO}_{2}$ are contacted with a liquid phase via distinct physical devices, a measure of the concentrations of said compound in the liquid phase is a reliable indication of the mass transfer capacity of each device. The variation of $\mathrm{pH}$ observed in the liquid phase when $\mathrm{CO}_{2}$ was supplied by plain bubbling, or by diffusion through the hydrophobic (HIFO) or the hydrophilic (HIFI) hollowfiber membranes, is depicted in Figure 4. It can be observed that the decrease in $\mathrm{pH}$ is larger in the liquid vessel connected to the hydrophobic membrane. Concomitantly, the increases in total inorganic carbon in the liquid phase, as calculated by the carbonic acid dissociation equilibrium relationships (Ferreira et al., 1998), are higher for the HIFO membrane, followed by bubbling and finally by the HIFI membrane (Figure 5). Equation 4 was fitted to these values, thus generating the corresponding overall volumetric coefficients of $\mathrm{CO}_{2}$ transfer $\left(\mathrm{K}_{\mathrm{L}} \mathrm{a}\right): 1.48$ $\times 10^{-2} \mathrm{~min}^{-1}$ for HIFO, $1.33 \times 10^{-2} \mathrm{~min}^{-1}$ for HIFI, and $7.0 \times 10^{-3} \mathrm{~min}^{-1}$ for bubbling. The estimates obtained for the various (related) coefficients are summarized in Table 1. As observed, the estimates of $K_{L} a$ for both membranes are above the estimate for bubbling (as expected, because of their higher available area for mass transfer), but surprisingly, both are of the same order of magnitude. Recalling that the liquid flow rate is the same

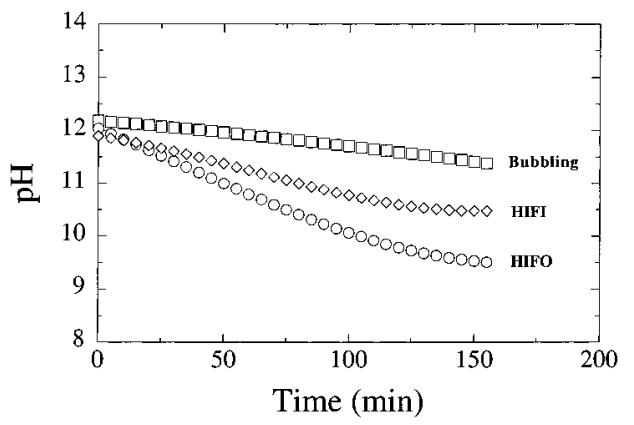

Figure 4. Evolution of $\mathrm{pH}$ during the experimental determinations of $\mathrm{K}_{\mathrm{L}} \mathrm{a}$ in the HIFO membrane, in the HIFI membrane, and by bubbling.

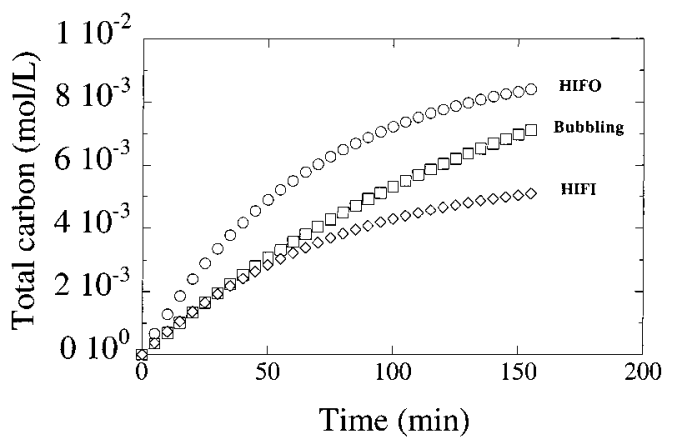

Figure 5. Evolution of the total carbon concentration during the experimental determinations of $K_{L} a$ in the HIFO membrane, in the HIFI membrane, and by bubbling.

in both systems, it thus seems that the number of fibers used (7400 in HIFO versus 140 in HIFI) does not significantly affect the mass transfer coefficient. This leads to the conclusion that there should be another limiting factor for mass transfer instead of availablearea of contact.

Since the available areas for mass transfer of the systems are quite different from each other $\left(1.7 \mathrm{~m}^{2}\right.$ for HIFO versus $1.4 \times 10^{-1} \mathrm{~m}^{2}$ for $\mathrm{HIFI}$, and $1.1 \times 10^{-3} \mathrm{~m}^{2}$ for bubbling; see Appendix), it is more meaningful to compare the values of the volumetric individual mass transfer coefficients $\left(\mathrm{K}_{\mathrm{L}}\right)$ for each system. Recall that the term a in $\mathrm{K}_{\mathrm{L}}$ a pertains to the gas-liquid interfacial area per unit volume of liquid, i.e., the ratio of the effective area (taking in account the porosity and pore pattern of each membrane) of mass transfer (A) to the liquid-phase volume $(\mathrm{V})$, which is equal to $1 \mathrm{~L}$ in all three cases. From the values for such specific area, it is then possible to determine the volumetric individual mass transfer coefficients, which are $1.45 \times 10^{-7} \mathrm{~m} / \mathrm{s}$ for HIFO, $1.59 \times 10^{-6}$ $\mathrm{m} / \mathrm{s}$ for HIFI, and $1.11 \times 10^{-4} \mathrm{~m} / \mathrm{s}$ for bubbling. It is interesting to notice that the highest value is associated with transfer by plain bubbling, i.e., the mass transfer flux is far more effective in bubbling. A possible explanation derives from the fully devel oped turbulent conditions produced during formation of the bubble (Malcata, 1991), which are essentially absent in the fibers. Owing to their reduced thickness, the burst pressure of the hollow-fibers is low, so their maximum operating pressure is generally below 2 bar. This upper limitation on the operational pressure restricts the maximum pressure drop and thus the flow rate along the fibers. As a result the regime is likely laminar (or transitional, at most), which obviously brings about low mass transfer coefficients; this situation is more dramatic in the HIFO fibers as a result of their scanty diameter ( $240 \mu \mathrm{m}$ versus $1000 \mu \mathrm{m}$ for the HIFI). Nevertheless, the high mass transfer area associated 
Table 1. Values for $K_{L}$ and $K_{L} a$ Using Several Transfer Devices

\begin{tabular}{clllll}
\hline transfer device & \multicolumn{1}{c}{$\mathrm{K}_{\mathrm{La}}\left(\mathrm{min}^{-1}\right)$} & $\mathrm{A}\left(\mathrm{m}^{2}\right)$ & $\mathrm{a}\left(\mathrm{m}^{-1}\right)$ & \multicolumn{1}{c}{$\mathrm{K}(\mathrm{m} / \mathrm{s})$} & reference \\
\hline HIFO & $1.48 \times 10^{-2}$ & 1.7 & 1700 & $1.45 \times 10^{-7}$ & present study \\
HIFI & $1.33 \times 10^{-2}$ & $1.4 \times 10^{-1}$ & 140 & $1.59 \times 10^{-6}$ & present study \\
HIFO & $(3.6-7.5) \times 10^{-3}$ & $2.38 \times 10^{-3}$ & 4.76 & $(1.26-2.64) \times 10^{-5}$ & Ferreira et al. (1998) \\
bubbling & $7.00 \times 10^{-3}$ & $1.1 \times 10^{-3}$ & 1.05 & $1.11 \times 10^{-4}$ & present study \\
bubbling & $(7.59-21.7) \times 10^{-2}$ & na & $2.15-6.21^{\mathrm{b}}$ & $(5.83-5.88) \times 10^{-4}$ & Talbot et al. (1991) \\
bubbling & $(9-94) \times 10^{-2}$ & na & $42.8-147.2^{\mathrm{b}}$ & $(3.51-10.6) \times 10^{-5}$ & Molina-Grima et al. (1993)
\end{tabular}

${ }^{\mathrm{a}}$ na $=$ not available. ${ }^{\mathrm{b}}$ Empirical values.

with the membranes smoothes out these differences in mass transfer coefficients.

Since the liquid boundary layer is assumed to dominate the mass transfer process in both the HIFO and HIFI systems, apparently there should be no differences in the $\mathrm{K}_{\mathrm{L}}$ for the two systems if the hydrodynamic conditions prevailing were similar. However, the HIFO system exhibits a $K_{L}$ value that is 1 order of magnitude lower than the HIFI counterpart; the explanation for this fact lies probably on the average linear velocity of liquid, which in the HIFI system is 3-fold that in the HIFO system, thus leading to the thinning of the boundary layer and consequently to a higher $\mathrm{K}_{\mathrm{L}}$. These observations also reinforce the assumption (previously taken as valid) that the mass transfer resistance associated with diffusion inside the HIFI pores of the membrane could be neglected, and that the mass transfer process in both membranes should be similar (because otherwise $K_{L}$ for HIFI should be lower than that for HIFO).

I deally, it would have been better to compare the two membranes under identical geometrical conditions (viz., inner diameter, and number and length of fibers), but there were no such membranes available in the market. Besides, scientific research encompassing systems available in the market is more prone to rel evance with regard to commercial applications.

In conclusion, the values of $\mathrm{K}_{\mathrm{L}}$ a for the HIFO and HIFI membranes are higher than those obtained for bubbling, so there seems to be some advantage in using the former devices.

It could be argued, since the interfacial areas are a rather important parameter in terms of observed mass transfer rates, that the system used to perform bubbling (i.e., a single, fairly large orifice) was not optimal when compared with the tiny pores of the membranes. Nevertheless, such bubbling systems have been classically preferred in microalgal cultures because of their increased turbulence, which in addition avoids biofouling on the bottom of the reactor.

Ferreira et al. (1998) tested a hydrophobic hollow-fiber device possessing a small transfer area $\left(2.38 \times 10^{-3} \mathrm{~m}^{2}\right)$, which consisted of a module containing only 10 fibers. The val ues reported for $K_{\perp}$ a (calculated according to the process followed here) were $3.6 \times 10^{-3}$ to $7.5 \times 10^{-3} \mathrm{~min}^{-1}$ (depending on the liquid flow rate) and hence were lower than those reported in the present work; however, the values reported by those authors were higher for $\mathrm{K}_{\mathrm{L}}$ (1.3 $\times 10^{-5}$ to $2.6 \times 10^{-5} \mathrm{~m} / \mathrm{s}$ ). This apparent discrepancy is explained (at least partially) by the following rationale: the higher liquid flow rates that those authors employed, i.e., $1.75 \times 10^{-6}$ to $6.41 \times 10^{-6} \mathrm{~m}^{3} / \mathrm{s}$ versus $5.4 \times 10^{-7}$ to $5.5 \times 10^{-7} \mathrm{~m}^{3} / \mathrm{s}$ in our study, will surely enhance gas exchange along the fibers between the liquid and gas phases, thus increasing their $K_{L}$ values. Regarding $K_{L} a$, similar comparisons cannot be performed because either the number of fibers or the liquid flow rates are different in the two systems. It is still interesting to notice that, although the number of fibers in the hydrophobic membrane modules decreased from ca. 7400 in our study to
10 in the case reported by Ferreira et al. (1998), the corresponding fractional decrease in $\mathrm{K}_{\mathrm{L}} \mathrm{a}$ was far more modest.

Talbot et al. (1991) obtained experimental values of $\mathrm{K} \mathrm{La}\left(\mathrm{O}_{2}\right)$ in liquid media characterized by viscosities similar to that of water, which were then converted into $\mathrm{K} \mathrm{La}\left(\mathrm{CO}_{2}\right)$ by the quotient of diffusivities of $\mathrm{CO}_{2}$ and $\mathrm{O}_{2}$. The values for $\mathrm{K}_{\mathrm{L}} \mathrm{a}\left(\mathrm{CO}_{2}\right)$ thus generated, regarding perforated plates, ranged from $7.59 \times 10^{-2}$ to $21.7 \times 10^{-2}$ $\min ^{-1}$, and the corresponding $\mathrm{K}_{\mathrm{L}}$ values (converted via an empirical estimate for a) varied between $5.83 \times 10^{-4}$ and $5.88 \times 10^{-4} \mathrm{~m} / \mathrm{s}$, depending on temperature. Owing to the large differences in a, i.e., $1.05 \mathrm{~m}^{-1}$ in our study and $2.15-6.21 \mathrm{~m}^{-1}$ in the study by Tal bot and co-workers, the $K_{L} a$ values determined by them are higher than those determined by us (i.e., $7.00 \times 10^{-3} \mathrm{~min}^{-1}$ ). Note that such differences in the values of a are also rel ated to the type of apparatus used for bubble formation; in our work, a glass tube with only one nozzle was employed for bubbling, whereas those authors used a perforated device. As expected, the values for $K_{L}$ are of the same order of magnitude as ours $\left(1.11 \times 10^{-4} \mathrm{~m} / \mathrm{s}\right)$. Molina-Grima et al. (1993) obtained experimental values for $K_{L}$ a ranging from $9.0 \times 10^{-2}$ to $94.0 \times 10^{-2} \mathrm{~min}^{-1}$ in a culture of microalgae inside a fermenter, at various impeller tip speeds. However, the extra turbulence promoted by the use of impellers does not seem to have altered the hydrodynamic pattern sufficiently to produce an effect upon the mass transfer coefficients. In fact, their $K_{L}$ values increased from $3.51 \times 10^{-5}$ up to $10.6 \times 10^{-5} \mathrm{~m} / \mathrm{s}$ when agitation was employed, and these figures are not above those obtained in our study in the absence of convective agitation. The diameter of the bubbles generated in their study was, in any case, small, and it is known that $\mathrm{K}_{\mathrm{L}}$ for bubbling increases with increasing bubble diameter.

Empirical Correlations. In an attempt to check the reasonability of our experimental values, they were compared with those using empirical correlations.

The bubble mean diameter $\left(\mathrm{d}_{\mathrm{B}}\right)$ can be calculated according to the following equation (Tal bot et al., 1991):

$$
\mathrm{d}_{\mathrm{B}}=0.0287 \mathrm{~d}_{\mathrm{o}}^{0.5} \mathrm{Re}_{0}^{0.33}
$$

where $d_{0}$ is the diameter of the bubbling nozzle; the Reynolds number in the nozzle $\left(\mathrm{Re}_{0}\right)$ is calculated from the ratio of the diameter of the bubbling nozzle, the speed of gas through said nozzle $\left(\mathrm{V}_{0}\right)$, and the density and viscosity of the gas ( $\rho_{\mathrm{g}}$ and $\mu_{\mathrm{g}}$, respectively), viz.,

$$
\operatorname{Re}_{\mathrm{o}}=\frac{\mathrm{d}_{\mathrm{o}} \mathrm{V}_{\mathrm{o}} \rho_{\mathrm{g}}}{\mu_{\mathrm{g}}}
$$

The nozzle diameter was $6 \mathrm{~mm}$, and the speed through the nozzle was calculated from the gas flow rate measured and the known area of the nozzle. The average diameter of the bubbles generated was ca. $7.4 \mathrm{~mm}$. 
The gas holdup $\left(\epsilon_{\mathrm{g}}\right)$ was calculated via the following equation (Talbot et al., 1991):

$$
\epsilon_{\mathrm{g}}=\frac{1}{2+\left(\frac{0.35}{v_{\mathrm{g}}}\right)\left(\frac{\rho_{\mathrm{l}} \tau}{72}\right)^{0.33}}
$$

where $\rho_{\mathrm{l}}$ is the density of the liquid, $v_{\mathrm{g}}$ is the superficial gas velocity (calculated from the gas flow rate and the area of the glass vessel recipient), and $\tau$ represents the surface tension at the gas/liquid interface. From these values, it is possible to obtain the interfacial area per unit volume, viz.,

$$
a=\frac{6 \epsilon_{g}}{d_{B}}
$$

It is interesting to note that, when the aforementioned value of $a$ is used to calculate $K_{L}$ starting from the experimental value of $K_{L} a$, one obtains $1.84 \times 10^{-4} \mathrm{~m} / \mathrm{s}$, which is close to the figure obtained upon division of the experimental value of $K_{\mathrm{L}} \mathrm{a}$ by the ratio of the volume of liquid to the transfer area as calculated in the Appendix (i.e., $1.11 \times 10^{-4} \mathrm{~m} / \mathrm{s}$ ).

The empirical equation proposed by Hughmark for individual bubbles is widely used and is recommended especially when the bubbles have large diameters (say above $2.5 \mathrm{~mm}$ ), which is our case; said equation reads

$$
\begin{aligned}
& \mathrm{K}_{\mathrm{L}}= \\
& \frac{\mathrm{D}_{\mathrm{CO}_{2}}}{\mathrm{~d}_{\mathrm{B}}}\left[2+0.061\left(\operatorname{Re}_{\mathrm{B}}\right)^{0.779}\left(\frac{\mu_{1}}{\rho_{1} \mathrm{D}_{\mathrm{CO}_{2}}}\right)^{0.546}\left(\frac{\mathrm{d}_{\mathrm{B}} \mathrm{g}^{0.33}}{\mathrm{D}_{\mathrm{CO}_{2}}{ }^{0.66}}\right)^{0.116}\right]
\end{aligned}
$$

where $\mathrm{D}_{\mathrm{CO}_{2}}$ denotes the diffusivity of $\mathrm{CO}_{2}$ in the liquid phase. The Reynolds number of the liquid $\left(\mathrm{Re}_{B}\right)$ depends on the bubble diameter, the density and viscosity of the liquid ( $\rho_{1}$ and $\mu_{1}$, respectively), and the rising velocity of a single bubble $\left(\mathrm{V}_{\mathrm{t}}\right)$, viz.,

$$
\operatorname{Re}_{\mathrm{B}}=\frac{\mathrm{d}_{\mathrm{B}} \mathrm{V}_{\mathrm{t}} \rho_{\mathrm{I}}}{\mu_{\mathrm{l}}}
$$

which is calculated through

$$
\mathrm{V}_{\mathrm{t}}=\sqrt{\frac{2 \tau}{\mathrm{d}_{\mathrm{B}} \rho_{1}}+\frac{\mathrm{gd}_{\mathrm{B}}}{2}}
$$

The $K_{L}$ value obtained from these correlations is 2.85 $\times 10^{-4} \mathrm{~s}^{-1}$, a value that is of the same order of magnitude as the experimental one.

The overall mass transfer coefficients calculated for $\mathrm{CO}_{2}$ transfer into the solution of sodium hydroxide can be applied to the culture of microalgae; in fact, if mass transfer is enhanced by the reaction between $\mathrm{CO}_{2}$ and $\mathrm{NaOH}$, it will al so be enhanced by the uptake of $\mathrm{CO}_{2}$ by the algal cells. In fact, several authors (e.g., Lee and Pirt, 1984) have claimed that the transfer of $\mathrm{CO}_{2}$ in microalgal cultures may be as much as $80 \%$ higher than the one observed for a purely physical absorption process.

Alga Culture Performance. The main problems associated with the use of hollow-fiber devices to grow and keep microorganisms alive derive from bi ofouling (Chen, 1995; Ferreira et al., 1998), which decreases the mass fluxes across the membrane. This difficulty did not occur in our experiments, probably as a result of the relatively low biomass concentrations attained, which is

\section{Bubbling}

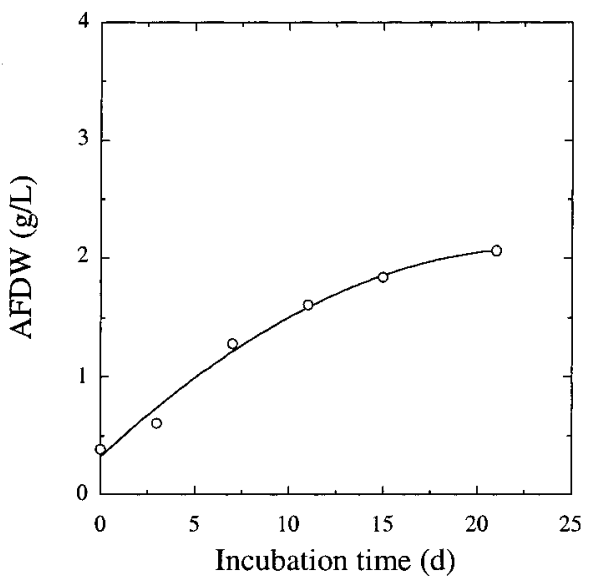

Membrane

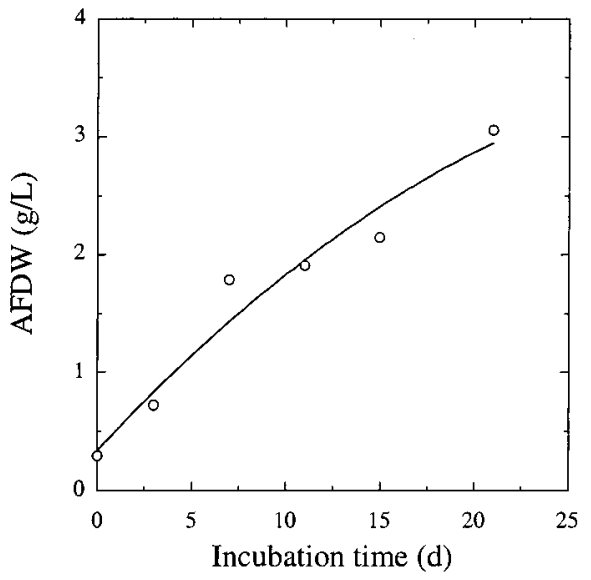

Figure 6. Evolution of ash-free dry weight (AFDW) during the experimental growth of $\mathrm{Nannochloropsis} \mathrm{sp.} \mathrm{with} \mathrm{bubbling} \mathrm{and}$ in the HIFO membrane at $1 \%(\mathrm{v} / \mathrm{v}) \mathrm{CO}_{2}$.

a common feature when dealing with cultures of microalgae (as opposed to yeast or bacteria). In our experiments, the microalgal culture was circulated through the lumen of the fibers in order to keep the tortuosity of the path to a minimum, a fact that may also have contributed to the absence of cell deposition and plugging (i.e., high tangential speeds).

The variation in the average AFDW throughout the incubation time for the systems tested is depicted in Figure 6. Since the performance of the algal cultures was essentially the same with every $\mathrm{CO}_{2}$ concentration and in both membranes, only one (typical) graph, for HIFO with $1 \%(\mathrm{v} / \mathrm{v}) \mathrm{CO}_{2}$, was depicted. The evolution of AFDW as time on stream elapsed shows a similar pattern for the control and the HIFO membrane devices; although the values for the cells cultivated with the membrane were slightly higher, the differences observed were not statistically significant. Hence, it can be concluded that the metabolic performance of cells, supplied with carbon dioxide by bubbling or by transmembrane diffusion, is essentially the same in terms of growth density.

Overall, the transfer of $\mathrm{CO}_{2}$ was not significantly enhanced by use of the hollow-fiber system. Other researchers (Ferreira et al., 1998) reached similar conclusions. When cultivating microalgal cells by circulating the culture through the lumen of the fibers, light/dark cycl es are imposed onto the culture. Since the microalga studied operates photosynthetically, it converts inorganic $\mathrm{CO}_{2}$ to organic carbon in the presence of light. In the 
Iumen of the fibers, cells have access to reasonable amounts of $\mathrm{CO}_{2}$, but the source of energy (i.e., light) is severely hampered. The residence times of the algal cells inside each fiber module were estimated (using the plug flow assumption) to be $235 \mathrm{~s}$ for HIFO and $81 \mathrm{~s}$ for HIFI. During these periods, algae are subject to darkness, so they will not intake $\mathrm{CO}_{2}$ from the surroundings; in fact, they are likely to excrete $\mathrm{CO}_{2}$ because of the respiration process. Therefore, a possible explanation for the lack of enhancement of growth of a photosynthetic microalga when the hollow-fiber devices were used, even in the presence of enhanced $\mathrm{CO}_{2}$ transfer, may derive from the fact that the periods of $\mathrm{CO}_{2}$ availability and light availability are out of phase. To illustrate this point, note that there are reports of measurable changes in $\mathrm{O}_{2}$ concentration within the first $4 \mathrm{~s}$ after switching off the light source in the case of cultures of cyanobacteria (Lassen et al., 1998).

Nevertheless, $K_{L}$ a values for the membranes are higher than those associated with bubbling, so there is a physicochemical gain, which unfortunately is not matched by a biotechnological gain. Even in this case, it should be emphasized that the use of hollow-fiber membrane reactors, owing to the indirect supply of $\mathrm{CO}_{2}$ to the cells, will not permit the concentrations of this gas in the medium to be high enough to become inhibitory (or even lethal) to the cells. Consequently, and according to the culture requirements, it is possible to recirculate pure $\mathrm{CO}_{2}$, thus avoiding the need for preliminary mixing of $\mathrm{CO}_{2}$ with air.

Although this indirect way of supplying $\mathrm{CO}_{2}$ by molecular transport has similarities with the process described by Kun-Lee and K wan-Hing (1989) with silicone tubing, our system configuration makes it possible to use lower gas pressures, as no need to counterbalance hydrostatic heads exists.

Optical microscopic observations were frequently performed in order to check for the integrity of the microalgal cells. It was observed that the circulation of the cells through the lumen of the hollow fibers did not cause disruption. This fact was anticipated because the internal diameter of the fibers is, in both cases, sufficiently large to accommodate ellipsoidal cells of ca. $3-10 \mu \mathrm{m}$ in diameter.

\section{Conclusions}

The use of microporous hollow fibers, rather than plain bubbling, for transfer of $\mathrm{CO}_{2}$ into microalgal cultures offers technological enhancements in effectiveness of mass transfer. The values for the parameter $K_{L} a$ are higher than those obtained by plain bubbling and, in addition, this type of system offers the opportunity to recirculate the gas and to use lower gas pressures, thus reducing operating costs. H owever, the overall metabolic enhancement in microalgal growth is negl igible, probably because $\mathrm{CO}_{2}$-rich and light-rich periods do not coincide with each other.

\section{Notation}

a

A

$A_{B}$

C

inorganic carbon concentration $\left(\mathrm{mol} / \mathrm{L}^{3}\right)$

$\mathrm{d}_{\mathrm{o}} \quad$ diameter of bubbling nozzle $(\mathrm{L})$

$\mathrm{d}_{\mathrm{B}} \quad$ average bubble diameter $(\mathrm{L})$

$\mathrm{D}_{\mathrm{CO}_{2}}$ diffusivity of $\mathrm{CO}_{2}$ in the liquid phase $\left(\mathrm{L}^{2} / \mathrm{T}\right)$
$D_{\text {eff }} \quad$ effective diffusivity within the porous membrane $\left(\mathrm{L}^{2} / \mathrm{T}\right)$

$D_{L} \quad$ diffusivity of $\mathrm{CO}_{2}$ in water $\left(\mathrm{L}^{2} / \mathrm{T}\right)$

g acceleration of gravity $\left(\mathrm{LT}^{2}\right)$

$g_{c} \quad$ conversion factor $\left(M L / \mathrm{FT}^{2}\right)$

$\mathrm{h} \quad$ height of glass vessel $(\mathrm{L})$

$\mathrm{H} \quad$ Henry's constant $\left(\mathrm{ML}^{2} / \mathrm{molT}^{2}\right)$

$\mathrm{k}_{\mathrm{g}} \quad$ gas-phase mass transfer coefficient (molT/ML)

$\mathrm{k}_{\mathrm{L}} \quad$ liquid-phase mass transfer coefficient $(\mathrm{L} / \mathrm{T})$

$\mathrm{k}_{\mathrm{m}} \quad$ transmembrane mass transfer coefficient (molT/ $M L)$

$\mathrm{K}_{\mathrm{L}} \quad$ volumetric overall mass transfer coefficient, based on the liquid concentrations $(\mathrm{L} / \mathrm{T})$

$\mathrm{n}_{\mathrm{B}} \quad$ number of bubbles produced in a certain period of time $\left(\mathrm{T}^{-1}\right)$

$\mathrm{p} \quad$ parameter (dimensionless)

$\mathrm{Q}_{\mathrm{g}} \quad$ gas flow rate $\left(\mathrm{L}^{3} / \mathrm{T}\right)$

$r_{B} \quad$ average bubble radius $(L)$

$r^{*} \quad$ parameter $(L)$

$\mathrm{Re} \quad$ Reynolds number (dimensionless)

$\mathrm{t} \quad$ time $(\mathrm{T})$

$t_{f} \quad$ time to generate one gas bubble (T)

$t_{s} \quad$ time for one bubble to reach the surface (T)

$\mathrm{V}^{*} \quad$ parameter (dimensionless)

$\mathrm{V} \quad$ liquid-phase volume $\left(\mathrm{L}^{3}\right)$

$\mathrm{V}_{\mathrm{O}} \quad$ velocity through nozzle $(\mathrm{L} / \mathrm{T})$

$\mathrm{V}_{\mathrm{t}} \quad$ rising velocity of a single bubble $(\mathrm{L} / \mathrm{T})$

$\delta \quad$ average radius of the pores of the membranes (L)

thickness of the stagnant liquid film (L)

$\epsilon_{\mathrm{g}} \quad$ gas holdup (dimensionless)

$\mu \quad$ viscosity (M/LT)

$\rho_{\mathrm{l}} \quad$ liquid density $\left(\mathrm{M} / \mathrm{L}^{3}\right)$

$\rho_{\mathrm{g}} \quad$ gas density $\left(\mathrm{M} / \mathrm{L}^{3}\right)$

$\tau \quad$ surface tension $\left(\mathrm{M} / \mathrm{T}^{2}\right)$

$v_{\mathrm{g}} \quad$ superficial gas velocity $(\mathrm{L} / \mathrm{T})$

$\xi \quad$ thickness of the membrane $(\mathrm{L})$

\section{Acknowledgment}

Financial support (grant BD/2838/93-IF) by PRAXIS XXI (Portugal) is hereby gratefully acknowledged. The authors are also grateful for the technical assistance by Dr. Victor Balcão in producing the scanning electron micrographs.

\section{Appendix}

Determination of the Total Transfer Area in the Plain Bubbling System. Consider that

$$
A=A_{B} \times \frac{n_{B}}{t} \times t_{S}
$$

where $A_{B}$ is the area available for mass transfer in one single bubble, $n_{B}$ is the number of bubbles produced in a certain period of time, $t_{s}$ is the time for one bubble to reach the free surface, and $A$ is the total transfer area in the plain bubbling system.

(a) Calculation of $A_{B}$. If

$$
t_{f}=\frac{191 \sqrt{Q_{g}^{1 / 5}}}{168 g^{3 / 5}} \quad(\text { Malcata, 1991) }
$$

where $t_{f}$ pertains to the time necessary to generate one 
gas bubble at the tip of the nozzle, and $Q_{g}$ is the gas flow rate, thus the average radius of a single bubble can be determined to be

$$
r=\sqrt[3]{\frac{3 Q_{g} t_{f}}{4 \Pi}}
$$

and the corresponding surface area is

$$
A_{B}=4 \Pi r^{2}
$$

(b) Determination of $\mathbf{t}_{\mathbf{s}}$. Since $1<\mathrm{d}_{\mathrm{B}}<10 \mathrm{~mm}$, then the rising velocity depends on the surface tension (Kay and Nedderman, 1985); hence, using

$$
\mathrm{p}=\frac{\tau^{3} \rho_{\mathrm{l}}}{\mathrm{g} \mu_{1}^{4^{\prime}}}, \quad \mathrm{r}^{*}=\frac{\mathrm{d}_{\mathrm{B}}}{2} \sqrt[3]{\frac{\rho_{1}^{2} \mathrm{~g}}{\mu_{1}^{2}}}
$$

and

$$
\mathrm{v}^{*}=\sqrt{2} \times \mathrm{p}^{1 / 12}
$$

it is possible to calculate the rising velocity of a single bubble, viz.,

$$
\mathrm{V}_{\mathrm{t}}=\frac{\mathrm{V}^{*}}{\sqrt[3]{\frac{\rho_{\mathrm{l}}}{\mu_{1} \mathrm{~g}}}} \text { (Kay and Nedderman, 1985) }
$$

where $\rho_{\mathrm{l}}$ and $\mu_{1}$ denote the density and viscosity of the liquid, respectively.

Finally, the time for one bubble to reach the surface can be obtained via

$$
\mathrm{t}_{\mathrm{s}}=\frac{\mathrm{h}}{\mathrm{v}_{\mathrm{t}}}
$$

where $\mathrm{h}$ is the height of the glass vessel.

Combining the above pieces of information, the area available for the gas-liquid transfer becomes, in our case, $1.1 \times 10^{-3} \mathrm{~m}^{2}$.

\section{References and Notes}

Becker, E. W. Large-Scale Cultivation. In Microalgae Biotechnology and Microbiology; Becker, E. W., Ed.; Cambridge University Press: UK, 1994; pp 63-171.

Borowitzka, M. A. Algal Growth Media and Sources of Algal Cultures. In Micro-algal Biotechnology; Borowitzka, M. A., Borowitzka, L. J ., Eds.; Cambridge University Press, New York, 1988; p 462.

Breck, W. G.; Brown, R. J . C.; McCowan, J . D. The Properties of Liquid Solutions. In Chemistry for Scienceand Engineering; Breck, W. G., Brown, R. J . C., McCowan, J . D., Eds.; McGrawHill International Editions: Singapore, 1989; pp 651-689.
Carvalho, A. P.; Malcata, F. X. Effect of Culture Media on Production of Polyunsaturated Fatty Acids by Pavlova lutheri. CryptogamieAlgol. 2000, 21, 59-71.

Chen, F.; J ohns, M. R. A Strategy for High Cell Density Culture of Heterotrophic Microalgae with Inhibitory Substrates. J. Appl. Phycol. 1995, 7, 43-46.

Ferreira, B. S.; Fernandes, H. L.; Reis, A.; Mateus, M. Microporous Hollow Fibres for Carbon Dioxide Absorption: Mass Transfer Model Fitting and the Supplying of Carbon Dioxide to Microalgal Cultures. Chem. Technol. Biotechnol. 1998, 71, 61-70.

Gallagher, S. L.; Tharakan, J . T.; Chau, P. C. An IntercalatedSpiral Wound Hollow Fiber Bioreactor for the Culture of Mammalian Cells. Biotechnol. Tech. 1987, 1, 91-96.

Goldman, J . C.; Dennett, M. R.; Riley, C. B. Inorganic Carbon Sources and Biomass Regulation in Intensive Microalgal Cultures. Biotechnol. Bioeng. 1981, 23, 995-1014.

Heussler, P.; Castillo, S. J .; Merino, M. F.; Vasquez, V. V. Improvements in Pond Construction and $\mathrm{CO}_{2}$ Supply for the Mass Production of Microal gae. Arch. Hydrobiol. Beih. Ergebn. Limnol. 1978, 11, 254-258.

Kay, J. M.; Nedderman, R. M. Two-Phase Flow. In Fluid Mechanics and Transfer Processes; Kay, J. M., Nedderman, R. M., Eds.; Cambridge University Press: UK, 1985; pp 499520.

Kun-Lee, $\mathrm{Y}$.; K wan-Hing, $\mathrm{H}$. Supplying $\mathrm{CO}_{2}$ to Photosynthetic Algal Cultures by Diffusion through Gas-Permeable Membranes. Appl. Microbiol. Biotechnol. 1989, 31, 298-301.

Lassen, C.; Glud, R. N.; Ramsing, N. B.; Revsbech, N. P. A Method to Improve the Spatial Resolution of Photosynthetic Rates Obtained by Oxygen Microsensors. J . Phycol . 1998, 34, 89-93.

Lee, Y. K.; Pirt, S. J . $\mathrm{CO}_{2}$ Absorption Rate in an Algal Culture: Effect of pH. J . Chem. Technol. Biotechnol. 1984, 34B, 2832.

Malcata, F. X. Simulation of the Mass Transfer Behaviour of Individual Gas Bubbles Undergoing F ast Chemical Stripping Using the J et Stream Model. I nt. J . Heat Mass Transfer 1991, 34, 559-572.

Markov, S. A.; Bazin, M. J .; Hall, D. O. Hydrogen Photoproduction and Carbon Dioxide Uptake by Immobilized Anabaena variabilis in a Hollow-Fiber Photobioreactor. Enzyme Microb. Technol. 1995, 17, 306-310.

Marsot, P.; Cembella, A. D.; Mouhri, K. Croissance de la Biomasse Azotée du Phaeodactylum tricornutum (Bacillariophyceae) en Culture Discontinue Dialysante et Non-Dialysante. Can. J . Microbiol. 1992, 38, 945-952.

Molina-Grima, E.; Sanchez-Perez, J. A.; Garcia-Camacho, F.; Robles-Medina, A. Gas-Liquid Transfer of Atmospheric $\mathrm{CO}_{2}$ in Microalgal Cultures. Chem. Technol. Biotechnol. 1993, 56, 329-337.

Talbot, P.; Gortares, M. P.; Lencki, R. W.; de la Noue, J . Absorption of $\mathrm{CO}_{2}$ in Algal Mass Culture Systems: A Different Characterization Approach. Bi otechnol. Bioeng. 1991, 37, 834-842.

Tapie, A.; Bernard, A. Microalgae Production: Technical and Economic Evaluations. Biotechnol. Bioeng. 1988, 32, 873885. 